\title{
A Data-Driven Intervention Framework for Improving Adherence to Growth Hormone Therapy Based on Clustering Analysis and Traffic Light Alerting Systems
}

\author{
Matheus ARAÚJO ${ }^{\mathrm{a}, 1}$, Paula VAN DOMMELEN ${ }^{\mathrm{b}}$, Jaideep SRIVASTAVA ${ }^{\mathrm{a}}$ and \\ Ekaterina KOLEDOVA ${ }^{\mathrm{c}}$ \\ ${ }^{a}$ Computer Science Department, University of Minnesota, Minneapolis, MN, USA \\ ${ }^{\mathrm{b}}$ The Netherlands Organization for Applied Scientific Research TNO, Leiden, \\ The Netherlands \\ ${ }^{c}$ Global Medical Affairs Cardiometabolic \& Endocrinology, \\ Merck Healthcare KGaA, Darmstadt, Germany
}

\begin{abstract}
Recombinant human growth hormone ( $\mathrm{r}-\mathrm{hGH})$ is an established therapy for growth hormone deficiency (GHD); yet, some patients fail to achieve their full height potential, with poor adherence and persistence with the prescribed regimen often a contributing factor. A data-driven clinical decision support system based on "traffic light" visualizations for adherence risk management of patients receiving $\mathrm{r}$ hGH treatment was developed. This research was feasible thanks to data-sharing agreements that allowed the creation of these models using real-world data of r-hGH adherence from easypod ${ }^{\mathrm{TM}}$ connect; data was retrieved for 11,015 children receiving r-hGH therapy for $\geq 180$ days. Patients' adherence to therapy was represented using four values (mean and standard deviation [SD] of daily adherence and hours to next injection). Cluster analysis was used to categorize adherence patterns using a Gaussian mixture model. Following a traffic lights-inspired visualization approach, the algorithm was set to generate three clusters: green, yellow, or red status, corresponding to high, medium, and low adherence, respectively. The area under the receiver operating characteristic curve (AUC-ROC) was used to find optimum thresholds for independent traffic lights according to each metric. The most appropriate traffic light used the SD of the hours to the next injection, with an AUCROC value of 0.85 when compared to the complex clustering algorithm. For the daily adherence-based traffic lights, optimum thresholds were $>0.82$ (SD, <0.37), $0.53-0.82$ (SD, 0.37-0.61), and $<0.53$ ( $\mathrm{SD},>0.61$ ) for high, medium, and low adherence, respectively. For hours to next injection, the corresponding optimum thresholds were $<27.18$ (SD, <10.06), 27.18-34.01 (SD, 10.06-29.63), and >34.01 (SD, >29.63). Our research indicates that implementation of a practical data-driven alert system based on recognised traffic-light coding would enable healthcare practitioners to monitor sub-optimally-adherent patients to $\mathrm{r}$-hGH treatment for early intervention to improve treatment outcomes.
\end{abstract}

Keywords. Adherence, recombinant human growth hormone, growth hormone deficiency, cluster modeling, pediatrics

\footnotetext{
${ }^{1}$ Corresponding author, Matheus Araújo, Computer Science Department, University of Minnesota,
} Minneapolis, MN 55455, USA; E-mail: arauj021@umn.edu.fauc 


\section{Introduction}

The use of r-hGH therapy to improve growth outcomes in children is well-established [1]. However, long-term studies have reported that patients often fail to achieve their full height potential [2], with sub-optimal adherence to r-hGH medication and poor persistence with the prescribed regimen considered major contributing factors $[1,3]$.

Historically, FAIR Principles [4] have relied upon clinical databases. However; there is potential to create new data-driven applications from patient-generated data [5]. In this study, we show how using the principle of responsible data-sharing [6] allowed creation of new innovations in data-driven applications for connected medical devices.

Digital health devices that monitor treatment adherence have the potential to improve patient/caregiver engagement and clinical outcomes [7]. For patients receiving r-hGH treatment (somatropin; Saizen ${ }^{\circledR}$, Merck Healthcare KGaA, Darmstadt, Germany), the easypod ${ }^{\mathrm{TM}}$ auto-injector device, in combination with easypod ${ }^{\mathrm{TM}}$ connect, allows automatic recording and real-time data transmission of the date, time, and dose injected [8] enabling healthcare professionals to monitor patient adherence and growth outcomes. Data from connected devices has also contributed to the development of machinelearning algorithms to predict adherence behavior in multiple therapy areas [9-11].

Traffic light visualizations can help to guide and improve clinical decisions through the use of an established association between colors and related therapy signals which have been applied in multiple therapy areas $[12,13]$. The concept of patient management through traffic light coding has also been successful in an emergency room setting, where the use of a three-tier urgency code helped prioritize patients for intervention [14].

\section{Methods}
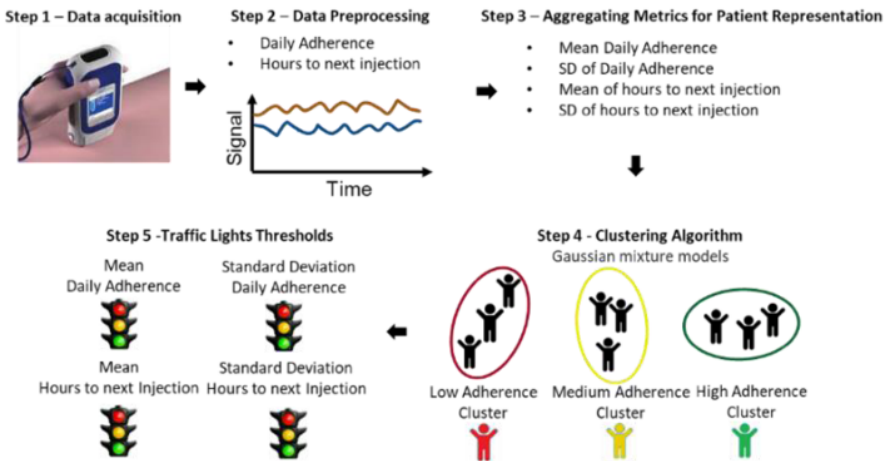

Figure 1. Methodological overview (with each step referred to hereafter).

Adherence data (date and time of injection, injected dose, prescribed dose) from easypod $^{\mathrm{TM}}$ connect were collected from 11,015 children receiving $\mathrm{r}-\mathrm{hGH}$ for $\geq 180$ days from January 2007-June 2019 (Figure 1, Step 1). Individual daily adherence was calculated: daily injected dose/daily prescribed dose (Figure 1, Step 2).

To determine injection consistency based on timing, 'hours to next injection' was computed using two daily signals (adherence and hours to next injection), aggregated with two metrics, the mean and SD of each patient during 180 days of r-hGH treatment. Patients' adherence to therapy was represented using a total of four values (mean and SD of adherence, and mean and SD of hours to next injection) (Figure 1, Step 3). 


\subsection{Model design and definition of adherence thresholds}

Cluster analysis was used to categorize adherence patterns using a Gaussian mixture model, implemented in the Python 3.7 library, scikit-learn 0.23.1 [15]. Patient data were normalized using a z-score normalization (Standard Scaler) for each metric. To define a traffic light-based system, three clusters were assigned in the clustering algorithm under the assumption that, if three patient groups were distinguishable, adherence patterns between clusters would be well-defined. Thus, each cluster should present a considerably different mean adherence level followed by different values of adherence SD and mean, and SD of hours to next injection (Figure 1, Step 4).

In total, four traffic lights were computed, one for each aggregated feature per patient, for which, two thresholds were used to define green, yellow, or red alert status corresponding to high, medium, and low adherence, respectively. To define the pair of thresholds (mean and SD) for each feature, we generated 10,000 synthetic samples from the fitted Gaussian distribution, representing patients that cover the model's feature space: 6,248, high adherence; 2,824, medium adherence; and 928, low adherence. For each feature/traffic light, 50 possible thresholds from values of the synthetic samples were randomly selected. All possible pair-wise combinations of thresholds were evaluated to define the three clusters previously defined by the more complex clustering algorithm. The best pair of thresholds represent when to signal green, yellow, and red for each feature. The area under the receiver operating characteristic curve (AUC-ROC) was used to determine optimum thresholds based on higher values (Figure 1, Step 5).

\section{Results}

The mean age of patients was 10.2 years (SD, 3.1 ); $58 \%$ were male and $42 \%$ female.

\subsection{Defined adherence clusters}

A Kruskal-Wallis H-Test was performed which ensured the metrics significantly differed across clusters $(\mathrm{p}<0.01)$. Cluster distribution is presented in Table 1 .

Table 1. Cluster centroids based on daily adherence and hours to next injection

\begin{tabular}{lcccc}
\hline \multirow{2}{*}{ Recorded Signal } & Aggregating & \multicolumn{3}{c}{ Cluster Centroids } \\
\cline { 3 - 5 } & Metric & $\begin{array}{c}\text { High } \\
(\mathbf{n}=\mathbf{6 8 1 0}, \mathbf{6 2} \%)\end{array}$ & $\begin{array}{c}\text { Medium } \\
(\mathbf{n}=\mathbf{3 1 8 6 , 2 9 \%})\end{array}$ & $\begin{array}{c}\text { Low } \\
(\mathbf{n}=\mathbf{1 0 1 9}, \mathbf{9 \%})\end{array}$ \\
\hline \multirow{2}{*}{ Daily adherence } & Mean & $0.92(0.07)$ & $0.72(0.13)$ & $0.46(0.32)$ \\
& Standard deviation & $0.32(0.17)$ & $0.48(0.10)$ & $0.47(0.21)$ \\
\multirow{2}{*}{ Hours to next injection } & Mean & $25.58(1.45)$ & $28.38(2.21)$ & $42.84(22.72)$ \\
& Standard deviation & $5.41(2.80)$ & $14.93(6.86)$ & $196.58(309.48)$ \\
\hline
\end{tabular}

\subsection{Traffic light thresholds}

The traffic light threshold values that best categorized patients according to high, medium, and low adherence for each metric are shown in Table 2. Patients with mean daily adherence of $>0.82$ trigger the green light, $0.53-0.82$ the yellow light, and $<0.53$ the red light. In another example, considering the mean of hours to next injection, the best thresholds were 27.18 and 34.01 , where values of $<27.18$ trigger the green light, 27.18-34.01 the yellow light, and $>34.01$ the red light. Table 2 also presents the AUC- 
ROC score to verify the performance of each traffic light while defining the three groups. The highest AUC-ROC value was 0.85 for the SD of the hours to next injection; thus, this is the traffic light that most resembles the prediction of the clustering algorithm.

Table 2. Traffic light thresholds and their corresponding discriminating capability (AUC-ROC)

\begin{tabular}{lccccc}
\hline \multirow{2}{*}{ Recorded Signal } & \multirow{2}{*}{$\begin{array}{c}\text { Aggregating } \\
\text { Metric }\end{array}$} & $\begin{array}{c}\text { Tow } \\
\text { (Red) }\end{array}$ & $\begin{array}{c}\text { Medium } \\
\text { (Yellow) }\end{array}$ & $\begin{array}{c}\text { High } \\
\text { (Green) }\end{array}$ & \multirow{2}{*}{ AUC-ROC } \\
\cline { 3 - 5 } & Mean & $<0.53$ & $0.53-0.82$ & $>0.82$ & 0.82 \\
\multirow{2}{*}{ Daily adherence } & Standard deviation & $>0.61$ & $0.37-0.61$ & $<0.37$ & 0.68 \\
& Mean & $>34.01$ & $27.18-34.01$ & $<27.18$ & 0.80 \\
\multirow{2}{*}{ Hours to next injection } & Standard deviation & $>29.63$ & $10.06-29.63$ & $<10.06$ & 0.85 \\
& & & & &
\end{tabular}

\section{Discussion}

This work is aligned with the need for further research into the visualization of datadriven applications [16]. Considering the cluster centroids detected by the model, higher SDs of both daily adherence and hours to next injection metrics were found in the lower adherence groups, suggesting that consistency is a key factor in distinguishing high and low adherence groups. Moreover, the high adherence group had an average mean daily usage of 0.92 (SD 0.07), reinforcing previous findings which classified $0.85 \%$ as the threshold defining good adherence to r-hGH therapy [3].

From the four proposed traffic lights, the SD of hours to next injection and mean daily adherence had a higher AUC-ROC. Thus, administering injections around the same time each day plays an essential role in maintaining high adherence to treatment and could serve as an important traffic light to alert clinicians to have discussions with patients/caregivers to mitigate the risk of sub-optimal adherence and, consequently, improve therapy effectiveness [2].

Study limitations include the small set of features used to create the traffic lights. Additional aggregating metrics, longitudinal data, and demographic information could be used but would also result in additional traffic lights. A filtering mechanism may be necessary, highlighting only the most useful traffic lights. It is recognized that other clustering algorithms (e.g. deep learning and time-series-based clustering algorithms) could be applied but with reduced model interpretability. Future work should evaluate whether the traffic lights would be meaningful for time frames beyond $\geq 180$ days.

Figure 2 shows the proposed application of the traffic lights, where three out of four traffic lights correspond to good therapy use. However, the patient data triggers a red light for 'Hours to next injection SD'; and investigation is strongly recommended.

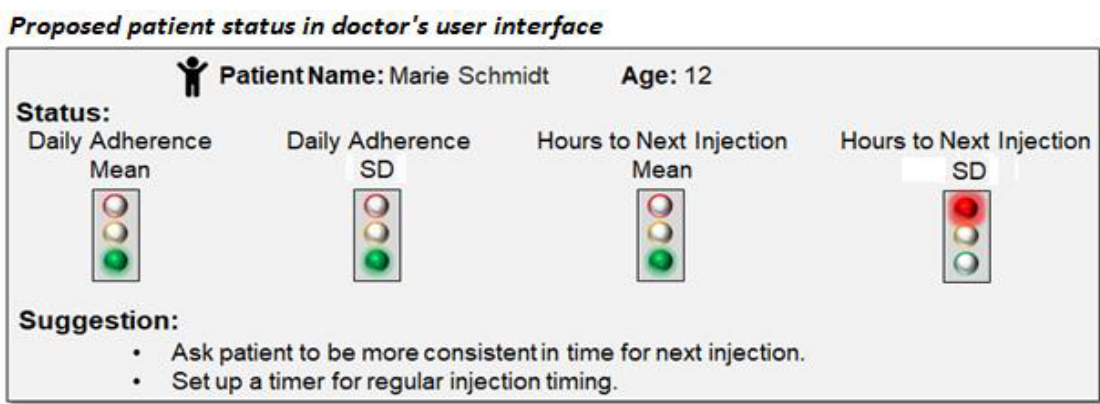

Figure 2. Fictional example showing applicability of traffic light-based thresholds. 


\section{Conclusions}

Our novel framework utilizes a clustering approach to categorize patients based on their $\mathrm{r}$-hGH therapy adherence levels, as determined from data from easypod ${ }^{\mathrm{TM}}$ connect. The proposed traffic light alerting system serves as a practical tool for therapy personalization to support optimal growth outcomes. Additional formative human factor studies and subsequent validation studies with healthcare professionals are warranted to understand how far the presented traffic light system might support clinical decision making.

\section{Disclosures}

This research was made possible thanks to a collaboration between The Netherlands Organization for Applied Scientific Research TNO, Leiden, The Netherlands \& Merck Healthcare KGaA, Darmstadt, Germany. MA, JS, and PvD have consultancy agreements with Merck. EK is an employee of Merck Healthcare KGaA, Darmstadt, Germany and holds shares in the company. Editorial assistance was provided by Sinéad Mutton, inScience Communications, Springer Healthcare Ltd, UK, funded by Merck Healthcare KGaA, Darmstadt, Germany.

\section{References}

[1] Graham S, et al. Identifying potentially modifiable factors associated with treatment non-adherence in paediatric growth hormone deficiency: A systematic review. Horm Res Paediatr. 2018;90(4):221-7.

[2] van Dommelen P, Koledova E, Wit JM. Effect of adherence to growth hormone treatment on 0-2 year catch-up growth in children with growth hormone deficiency. PLoS One. 2018;13(10):e0206009.

[3] Cutfield WS, Derraik JG, Gunn AJ, Reid K, Delany T, Robinson E, et al. Non-compliance with growth hormone treatment in children is common and impairs linear growth. PLoS One. 2011 Jan 31;6(1):e16223.

[4] Wilkinson MD, Dumontier M, Aalbersberg IJ, Appleton G, Axton M, Baak A, et al. The FAIR Guiding Principles for scientific data management and stewardship. Sci Data. 2016 Mar 15;3:160018.

[5] Sayeed R, Gottlieb D, Mandl KD. SMART Markers: Collecting patient-generated health data as a standardized property of health information technology. NPJ Digit Med. 2020;3:9.

[6] Summary of Merck's responsible data sharing policy: https://www.merckgroup.com/research/healthcare/Responsible-Data-Sharing-Policy-EN.pdf.

[7] Bittner B, Schmit Chiesi C, et al. Connected drug delivery devices to complement drug treatments: potential to facilitate disease management in home setting. Med Devices (Auckl). 2019;12:101-27.

[8] Koledova E, Stoyanov G, Ovbude L, Davies PSW. Adherence and long-term growth outcomes: results from the easypod ${ }^{\mathrm{TM}}$ connect observational study (ECOS) in paediatric patients with growth disorders. Endocr Connect. 2018 Aug;7(8):914-23.

[9] Araujo A, Kazaglis L, Iber C, Srivastava J. A data-driven approach for continuous adherence predictions in sleep apnea therapy management, 2019 IEEE International Conference on Big Data, pp. 2716-25.

[10] Araujo M, van Dommelen P, et al. Using Deep Learning for Individual-Level Predictions of Adherence with Growth Hormone Therapy. Stud Health Technol Inform. 2021 May 27;281:133-7.

[11] Tibble H, Chan A, Mitchell EA, Horne E, Doudesis D, Horne R, et al. A data-driven typology of asthma medication adherence using cluster analysis. Sci Rep. 2020 Sep 14;10(1):14999.

[12] Dagliati A, Sacchi L, Tibollo V, Cogni G, Teliti M, Martinez-Millana A, et al. A dashboard-based system for supporting diabetes care. J Am Med Inform Assoc. 2018 May 1;25(5):538-47.

[13] Saposnik G, Grueschow M, Oh J, Terzaghi MA, Kostyrko P, Vaidyanathan S, et al. Effect of an educational intervention on therapeutic inertia in neurologists with expertise in multiple sclerosis: A randomized clinical trial. JAMA Netw Open. 2020 Dec 16;3(12).

[14] Leppaniemi A, Jousela I. A traffic-light coding system to organize emergency surgery across surgical disciplines. Br J Surg. 2014 Jan;101(1):e134-40.

[15] Pedregosa F, Varoquaux G, Gramfort A, Michel V, Thirion B, Grisel O, et al. Scikit-learn: Machine Learning in Python. J Mach Learn Res. 2011;12:2825-30.

[16] West VL, Borland D, Hammond WE. Innovative information visualization of electronic health record data: a systematic review. J Am Med Inform Assoc. 2015 Mar;22(2):330-9. 